\title{
Effect of hyperbaric oxygen and vitamin $C$ and $E$ supplementation on biomarkers of oxidative stress in healthy men
}

\author{
Nicolle Bader ${ }^{1}$, Anja Bosy-Westphal ${ }^{2}$, Andreas Koch ${ }^{3}$, Gerald Rimbach ${ }^{4}$, Allan Weimann ${ }^{5}$, \\ Henrik E. Poulsen ${ }^{5}$ and Manfred J. Müller ${ }^{2}$ \\ ${ }^{1}$ Insititute of Biological Chemistry and Nutrition, University of Hohenheim, Garbenstrasse 28, D-70593 Stuttgart, Germany \\ ${ }^{2}$ Insititute of Human Nutrition and Food Science, Christian-Albrechts-Universität zu Kiel, Düsternbrooker Weg 17, \\ D-24105 Kiel, Germany \\ ${ }^{3}$ German Naval Medical Institute, Kopperpahler Allee 120, D-24119 Kronshagen, Germany \\ ${ }^{4}$ Insititute of Human Nutrition and Food Science, Christian-Albrechts-Universität zu Kiel, Hermann-Rodewald-Strasse 6, \\ D-24098 Kiel, Germany \\ ${ }^{5}$ Department of Clinical Pharmacology Q7642, Rigshospitalet, 9 Blegdamsvej, DK-2100 Copenhagen, Denmark
}

(Received 5 August 2006 - Revised 25 February 2007 - Accepted 15 March 2007)

\begin{abstract}
The objectives of the present study were to evaluate the effect of normobaric and hyperbaric $\mathrm{O}_{2}(\mathrm{HBO})$ on plasma antioxidants and biomarkers of oxidative stress in plasma and urine and to investigate the effect of a 4-week vitamin C plus E supplementation on HBO-induced oxidative stress. Nineteen healthy men were exposed to $\mathrm{HBO}\left(100 \% \mathrm{O}_{2} ; 240 \mathrm{kPa}\right)$ before and after 4 weeks' supplementation with $500 \mathrm{mg}$ vitamin $\mathrm{C}$ plus $165 \mathrm{mg}$ $\alpha$-tocopherol equivalents. Exposure to $21 \% \mathrm{O}_{2}$ at $100 \mathrm{kPa}$ served as intra-individual controls (control). Samples for the analysis of plasma antioxidants and oxidative stress biomarkers were collected before and immediately after each treatment. The present results showed that when compared with 'control', a single exposure to HBO resulted in a decrease of plasma vitamin $\mathrm{C}(P=0 \cdot 027)$ and an increase of lipid peroxides $(P=0.0008)$ and urinary 8-oxo-deoxyguanosine (8-oxodG) excretion $(P=0 \cdot 006)$. Oxidative stress was not prevented by a 4-week supplementation with vitamins $\mathrm{C}$ and $\mathrm{E}$. HBO-induced changes in plasma parameters correlated with basal antioxidant levels. The increase of urinary 8-oxodG after HBO plus supplementation correlated negatively with vitamin $\mathrm{E}$ intake $(P=0.023)$. We concluded that in healthy men HBO caused oxidative stress, which could not be prevented by dietary vitamin $\mathrm{C}$ plus E supplementation. The present data support the idea that HBO is a suitable model for oxidative stress in healthy volunteers.
\end{abstract}

Hyperbaric oxygen: Normobaric oxygen: Oxidative stress: Supplementation: Vitamin C: Vitamin E

The human organism is constantly exposed to oxidants (reactive oxygen species) from both physiological processes and pathophysiological conditions, foreign compound metabolism and radiation ${ }^{1}$. An increased production of reactive oxygen species together with a failure of the network of enzymic, endogenous and nutritional antioxidants leads to oxidative stress. Chronic oxidative stress may be involved in the development of chronic diseases such as cancer, CHD, neurodegenerative diseases, diabetes mellitus and cataract, and has also been suggested as a mechanism of ageing ${ }^{2}$. Therefore, maintaining the endogenous antioxidant defence system by supplementing with antioxidants appears worthwhile.

However, current research results concerning the protective effects of antioxidants on biomarkers of oxidative stress and on diseases are contradictory ${ }^{3-11}$. Several intervention studies showed some protective effects, while others did not. The results of a meta-analysis of nineteen randomised, placebo-controlled trials with high-dose vitamin $\mathrm{E}$ rather than dietary levels showed a dose-dependent relationship between vitamin E supplementation and all-cause mortality ${ }^{12}$. Specifically, all-cause mortality in volunteers with a high risk for a chronic disease progressively increased for dosages approximately greater than $100 \mathrm{mg} / \mathrm{d}$.

It has been suggested that a preventive effect of antioxidants may only be seen in a situation of high oxidative stress ${ }^{13}$ and we therefore based the present study on the following two concepts. First, a standardised and well-proven model of oxidative stress (hyperbaric $\mathrm{O}_{2}$; $\mathrm{HBO}$ ) was used to study the effects of an enhanced formation of reactive oxygen species using various parameters of oxidative stress. Second, intraand inter-individual variabilities of the biomarkers were assessed for comparison with the effects of HBO. HBO was also used to investigate the effect of vitamin $\mathrm{C}$ plus $\mathrm{E}$

Abbreviations: GSH, reduced glutathione; HBO, hyperbaric $\mathrm{O}_{2}$; NBO, normobaric $\mathrm{O}_{2}$; 8-oxodG, 8-oxo-deoxyguanosine; T0, control treatment; T1, 100\% oxygen under normobaric conditions; T2, $100 \%$ oxygen under hyperbaric conditions; T3, vitamin supplementation plus $100 \%$ oxygen under hyperbaric conditions.

* Corresponding author: Dr Nicolle Bader, fax +490711 45923386, email nbader@uni-hohenheim.de 
supplementation. Apparently healthy male non-smokers were examined, and to avoid the potential confounding factor of diet their antioxidant intake was also monitored.

\section{Materials and methods}

\section{Volunteers}

The study protocol was approved by the ethical committee of the Christian-Albrechts University of Kiel (Germany). All volunteers gave their written informed consent.

Nineteen men were recruited by notice board postings (age range 21-39 years). All volunteers were non-smokers and not taking any medication or vitamin supplementation. Exclusion criteria were claustrophobia and previous dives with 'nitrox' (breathing gas with a higher proportion of $\mathrm{O}_{2}$ than the normal $21 \%$ ). Volunteers were asked to retain their usual lifestyle and not to diet during the study period.

\section{Study protocol}

The study consisted of four protocols, referred to as T0, T1, T2 and T3, which were carried out over a period of 5 months (Fig. 1). The pressure chamber system Hydra $2000^{\circledR}$ (Haux, Karlsbad, Germany) was used for all treatments. Treatment with normobaric ambient air $(100 \mathrm{kPa}$ or $1.0 \mathrm{bar} ; 21 \%$ $\mathrm{O}_{2}$ ) served as the control treatment (T0). At T1 volunteers were treated with $100 \% \mathrm{O}_{2}$ under normobaric conditions (normobaric $\mathrm{O}_{2}$; NBO). At T2 and T3 volunteers were exposed to HBO $\left(240 \mathrm{kPa}\right.$ or $\left.2.4 \mathrm{bar} ; 100 \% \mathrm{O}_{2}\right)$. Each treatment consisted of $131 \mathrm{~min}$ and was interspersed with $2 \times 10 \mathrm{~min}$ periods of breathing ambient air $\left(21 \% \mathrm{O}_{2}\right)$. For $28 \mathrm{~d}$ before T3 volunteers were supplemented with a daily dose of $500 \mathrm{mg}$ slowrelease vitamin $\mathrm{C}$ and $182 \mathrm{mg} R R R$ - $\alpha$-tocopheryl acetate (corresponding to $165 \mathrm{mg} \alpha$-tocopherol equivalents; CellaVie; Ferrosan A/S, Soeborg, Denmark). Compliance was assessed by counting remaining pills and checking plasma levels of vitamin $\mathrm{C}$ and vitamin $\mathrm{E}$ before and after supplementation. Data from one subject were omitted because of a lack of compliance as evidenced by unchanged vitamin $\mathrm{C}$ and vitamin $\mathrm{E}$ levels after supplementation.

\section{Nutrient and fruit and vegetable intake}

Seven-day dietary records were analysed using the computer software Prodi 4.5 (Wissenschaftliche Verlagsgesellschaft $\mathrm{mbH}$, Stuttgart, Germany) at each study period. Number and size of daily portions of fruit and vegetables including fruit and vegetable juices were assessed according to the definitions of the worldwide campaign '5-a-day'.

\section{Sample collection}

Venous blood and urine samples were collected before and immediately after treatments at T0, T1, T2 and T3. Blood samples were immediately centrifuged at $2500 \mathrm{~g}$. Plasma and whole-blood fractions were stored at $-80^{\circ} \mathrm{C}$ until analyses. Urine samples were fumigated with $\mathrm{N}_{2}$ and immediately stored at $-80^{\circ} \mathrm{C}$ until 8-oxo-deoxyguanosine (8-oxodG) analysis.

\section{Measurement of antioxidants and antioxidant capacity}

Plasma levels of vitamin $C, \alpha$-tocopherol, retinol, $\beta$-carotene and reduced glutathione (GSH) in whole blood were measured by commercial HPLC kits supplied by Chromsystems (Munich, Germany). The preparation of the samples was done according to the manufacturer's description. Separation of antioxidants on a C18 column was followed by UV (vita$\min$ C, $245 \mathrm{~nm}$; $\alpha$-tocopherol, $295 \mathrm{~nm}$; retinol, $325 \mathrm{~nm}$; $\beta$-carotene, $453 \mathrm{~nm}$ ) and fluorescence detection (GSH, 385 and $510 \mathrm{~nm}$ ). A Waters HPLC system (Waters, Eschborn, Germany) with a pump (model 515; Waters), an autosampler (model 717plus; Waters), a UV detector (model 2487; Waters) and a fluorescence detector (model 1100; Hewlett Packard, Böblingen, Germany) was used. A commercial ELISA kit by Immundiagnostik (Bensheim, Germany) was used to measure plasma antioxidant capacity. Quantification of antioxidants was based on the reaction of exogenous peroxide with antioxidants present in the sample. Unreactive peroxides were quantified by a peroxidase-catalysed reaction. The reaction was stopped by adding acid to give a colorimetric endpoint that was read spectrophotometrically at $450 \mathrm{~nm}$ by the microplate reader Sunrise (Tecan, Crailsheim, Germany). Quantification was done by external calibration. The preparation of samples was done according to the manufacturer's description. Control samples were run together with each batch of samples. All measurements were done in duplicates.

\section{Spot urine 8-oxo-deoxyguanosine analysis}

Urinary 8-oxodG was measured by a previously described LCMS/MS method ${ }^{14}$. Briefly, frozen urine samples were thawed and diluted 1:1 with $100 \mathrm{~mm}$-lithium acetate buffer containing 8-oxodG labelled with stable isotopes. Samples were heated to $37^{\circ} \mathrm{C}$ for $10 \mathrm{~min}$ and whirly mixed before centrifugation at $5000 \mathrm{~g}$ for $10 \mathrm{~min}$. A Perkin Elmer Series 200 HPLC equipped with two pumps, autosampler, solvent cabinet and vacuum degasser was used (Perkin Elmer, Norwalk, CT, USA). The HPLC was fully controlled by the mass spectrometer (Sciex API 3000 triple quadrupole mass spectrometer) with a turboionspray source (Sciex, Thornill, Canada). The HPLC separation was performed on a Phenomenex Prodigy ODS HPLC

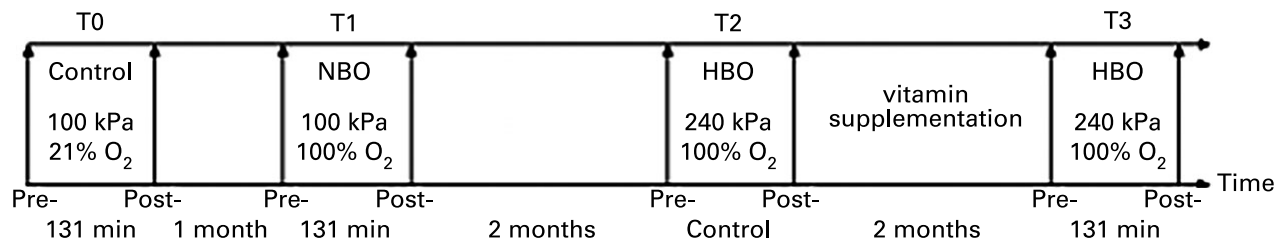

Fig. 1. Study protocol. NBO, normobaric $\mathrm{O}_{2} ; \mathrm{HBO}$, hyperbaric $\mathrm{O}_{2}$. 
column $(100 \times 2 \mathrm{~mm} ; 3 \mu \mathrm{m})$, protected by a C18 guard column $(4 \times 2 \mathrm{~mm})$, both obtained from Phenomenex (Torrance, CA, USA). The measurements were done in duplicates. Results are expressed as $\mathrm{ng} / \mathrm{mg}$ creatinine. Creatinine was measured by the Jaffé method ${ }^{15}$.

\section{Measurement of lipid peroxidation}

Malondialdehyde in plasma was measured by a commercial HPLC kit (Chromsystems, Munich, Germany). Samples were prepared accordingly to the manufacturer's description. The HPLC system consisted of a pump (model 2150; Pharmacia Biosystems, Freiburg, Germany), an autosampler (model AS-100; BioRad, Munich, Germany) and a fluorescence detector (model 1100; Hewlett Packard, Böblingen, Germany). Plasma lipid peroxides were measured photometrically with a commercial ELISA kit (Immundiagnostik, Bensheim, Germany) on a plate reader (Sunrise; Tecan, Crailsheim, Germany). Measurement of lipid peroxides was based on the reaction of peroxidase with peroxide and a subsequent reaction with tetramethylbenzidine. The reaction was stopped by adding acid to give a colorimetric endpoint that was read photometrically at $450 \mathrm{~nm}$. Quantification was done by external calibration. Control samples were run together with each batch of duplicate samples.

\section{Statistical analysis}

The power calculation was based on the variability of 8oxodG measurements obtained previously, a 0.05 type I error and a statistical power of $80 \%$. We calculated that a study size of nineteen participants would enable us to detect a change of $10 \%$ in 8 -oxodG excretion. Data were analysed using Statistica 6 (StatSoft, Tulsa, OK, USA) and are represented as mean values with their standard errors or medians and lower and upper quartiles as given in each table and figure. All data were tested for normality of distribution with the Shapiro-Wilk test. Due to a majority of non-parametric data, statistical analyses were performed by non-parametric tests.

Friedman's test was used for multiple statistical comparisons between the changes (pre-treatment $v$. post-treatment) of the treatments where subject is regarded as a random effect with treatment (T0, T1, T2, T3) as a fixed effect. The same test was used to compare baseline values with subject as a random effect and time (T0, T1, T2, T3) as a fixed effect. When the effect of treatment (or time) was significant, comparisons of treatment means (baseline means) were done by Wilcoxon's matched-pair signed-rank test. Spearman's correlation coefficient was used for relationships between different parameters. Intra- and inter-individual CV were calculated for all parameters assessed using the basal values at T0, T1 and T2. A 'sensitive' subject was defined as an individual whose response to $\mathrm{HBO}$ exceeded twice the square root of the within-subject variance. McNemar's test was performed to test significant changes in terms of 'sensitivity' in response between T2 and T3. $P$ values less than 0.05 were considered statistically significant.

\section{Results \\ Baseline data}

Basal anthropometric data, plasma antioxidant concentrations, biomarkers of oxidative damage and dietary intake of the volunteers at T0, T1, T2 and T3 are given in Table 1. There were no differences in dietary intake data between $\mathrm{T} 0, \mathrm{~T} 1, \mathrm{~T} 2$ and T3, respectively. These anthropometric data differed significantly between $\mathrm{T} 1$ and $\mathrm{T} 2(P<0 \cdot 05)$. At $\mathrm{T} 2$ plasma vitamin $\mathrm{C}$ concentration was higher when compared with $\mathrm{T} 0$ and $\mathrm{T} 1$ and vitamin $\mathrm{E}$ concentration increased from $\mathrm{T} 0$ to $\mathrm{T} 1$ $(P<0 \cdot 05)$. GSH concentrations at $\mathrm{T} 1$ and $\mathrm{T} 2$ were significantly higher compared with T0 $(P<0 \cdot 05)$. From the basal data at T0, T1 and T2 intra- and inter-individual CV were calculated for weight, BMI, plasma antioxidant concentrations, biomarkers of oxidative damage and nutrient intake, respectively (Table 1). Mean intra- and inter-individual $\mathrm{CV}$ ranged from 1.1 and $12.7 \%$ for BMI up to 76.9 and $112.1 \%$ for alcohol intake, respectively. In general the highest intra-individual and inter-individual variations were seen in variables of nutrient intake.

\section{Effects of control treatment, $100 \%$ oxygen under normobaric conditions and $100 \%$ oxygen under hyperbaric conditions}

Treatment with ambient air at $100 \mathrm{kPa}$ did not lead to changes (pre-T0 $v$. post-T0) in the concentrations of plasma antioxidants and biomarkers of oxidative damage $(P>0.05)$. In contrast, NBO resulted in a significant decrease of plasma vitamin $\mathrm{C}$ concentration when compared with the change at T0 (change in pre-T0-post-T0 $v$. change in pre-T1-post-T1; Table 2). HBO resulted in a significant decrease in plasma vitamin $\mathrm{C}$ concentration and also resulted in an increased urinary 8-oxodG excretion and increased plasma lipid peroxides in comparison with the control situation (change in pre-T0-postT0 v. change in pre-T2-post-T2; Table 2). However, HBOinduced changes were similar to the changes at NBO (change in pre-T1-post-T1 $v$. change in pre-T2-post-T2; $P>0.05)$. Taking into account the within-subject variation of the considered parameters, 'sensitive' and 'non-sensitive' volunteers can be differentiated (i.e. 'sensitive' volunteers are characterised by HBO-induced changes exceeding twice the square root of the within-subject variance) using lipid peroxides and urinary 8-oxodG as suitable biomarkers. From these data it was calculated that $58 \%$ (concerning lipid peroxides) and $72 \%$ of the volunteers (concerning urinary 8-oxodG) can be considered as 'sensitive' individuals at T2, respectively.

\section{Effect of vitamin intervention}

A 4-week supplementation with vitamin $\mathrm{C}$ and vitamin $\mathrm{E}$ significantly increased vitamin $\mathrm{C}$ and $\mathrm{E}$ plasma concentrations (pre-T3) when compared with the concentrations pre-T0, pre-T1 and pre-T2 $(P<0.05$; Fig. 2). Pre-T3 levels of GSH were lower when compared with basal data at T0, T1 and T2 $(P<0 \cdot 05$; Fig. 2). Also basal lipid peroxide levels significantly decreased after supplementation with vitamins $\mathrm{C}$ and $\mathrm{E}$ (148 v. $85 \mu \mathrm{mol} / \mathrm{l} ; P=0.002$; Table 1). 
Table 1. Anthropometrics, plasma antioxidant concentration, biomarkers of oxidative damage and nutrient intake of the study group at pre-T0, pre-T1, pre-T2 and pre-T3* (Medians and lower and upper quartiles)

\begin{tabular}{|c|c|c|c|c|c|c|c|c|c|c|c|}
\hline & \multicolumn{8}{|c|}{ Basal concentrations } & \multirow[b]{3}{*}{$\mathrm{CV}_{\text {intra }}(\%)$} & \multirow[b]{3}{*}{$\mathrm{CV}_{\text {inter }}(\%)$} & \multirow[b]{3}{*}{$\mathrm{CV}_{\text {intra }} / \mathrm{CV}_{\text {inter }}$} \\
\hline & \multicolumn{2}{|c|}{ T0 $(n 19)$} & \multicolumn{2}{|c|}{$\mathrm{T} 1(n 19)$} & \multicolumn{2}{|c|}{ T2 ( $n$ 19) } & \multicolumn{2}{|c|}{ T3 $(n 18)$} & & & \\
\hline & Median & LQ, UQ & Median & LQ, UQ & Median & LQ, UQ & Median & $\mathrm{LQ}, \cup \mathrm{U}$ & & & \\
\hline Weight (kg) & $79 \cdot 4^{\mathrm{a}, \mathrm{b}}$ & $72 \cdot 7,86 \cdot 3$ & $79 \cdot 3^{\mathrm{a}}$ & $72 \cdot 3,87 \cdot 0$ & $79 \cdot 8^{\mathrm{b}}$ & $73 \cdot 0,89 \cdot 5$ & $79 \cdot 7^{\mathrm{a}, \mathrm{b}}$ & $73 \cdot 1,89 \cdot 7$ & 1.6 & $14 \cdot 0$ & 0.11 \\
\hline BMI $\left(\mathrm{kg} / \mathrm{m}^{2}\right)$ & $24 \cdot 2^{a, b}$ & $21 \cdot 4,27 \cdot 2$ & $24 \cdot 1^{\mathrm{a}}$ & $21 \cdot 7,26 \cdot 9$ & $24 \cdot 3^{\mathrm{b}}$ & $21 \cdot 9,27 \cdot 1$ & $23 \cdot 9^{a, b}$ & $22 \cdot 2,25 \cdot 5$ & 1.1 & $12 \cdot 7$ & 0.09 \\
\hline \multicolumn{12}{|c|}{ Plasma antioxidant concentrations } \\
\hline Vitamin C $(\mu \mathrm{mol} / \mathrm{l})$ & $66 \cdot 0^{\mathrm{a}}$ & $47 \cdot 7,74 \cdot 6$ & $61 \cdot 5^{\mathrm{a}}$ & $54 \cdot 3,71 \cdot 7$ & $77 \cdot 1^{\mathrm{b}}$ & $63 \cdot 9,90 \cdot 0$ & $98.9^{\mathrm{C}}$ & $85 \cdot 3,104 \cdot 1$ & 11.4 & $27 \cdot 6$ & 0.41 \\
\hline Vitamin E $(\mu \mathrm{mol} / /)$ & $30 \cdot 0^{\mathrm{a}}$ & $26 \cdot 6,32 \cdot 0$ & $33 \cdot 1^{\mathrm{b}}$ & $29 \cdot 0,37.0$ & $31 \cdot 2^{a, b}$ & $26 \cdot 7,35 \cdot 4$ & $44 \cdot 4^{\mathrm{c}}$ & $42 \cdot 6,51 \cdot 5$ & $15 \cdot 0$ & 20.9 & 0.72 \\
\hline Vitamin A $(\mu \mathrm{mol} / \mathrm{l})$ & $2 \cdot 9$ & $2 \cdot 6,3 \cdot 4$ & 3.0 & $2 \cdot 8,3.5$ & 3.1 & $2 \cdot 6,4 \cdot 0$ & $3 \cdot 2$ & $2 \cdot 7,3 \cdot 6$ & $15 \cdot 8$ & $21 \cdot 3$ & 0.74 \\
\hline$\beta$-Carotene $(\mu \mathrm{mol} / \mathrm{l})$ & $311^{\mathrm{a}}$ & 181,400 & $295^{a}$ & 219,677 & $280^{\mathrm{a}}$ & 161,648 & $304^{\mathrm{b}}$ & 141,397 & $23 \cdot 6$ & 66.5 & 0.35 \\
\hline $\mathrm{AOC}(\mu \mathrm{mol} / \mathrm{l})$ & 228 & 219,243 & 241 & 233, 262 & 259 & 234,287 & 249 & 225,273 & 9.6 & $27 \cdot 2$ & 0.73 \\
\hline GSH $(\mu \mathrm{mol} / \mathrm{l})$ & $404^{\mathrm{a}}$ & 338,548 & $541^{b}$ & 462,667 & $523^{b}$ & 435,588 & $311^{c}$ & 277,361 & $22 \cdot 8$ & $27 \cdot 2$ & 0.84 \\
\hline \multicolumn{12}{|c|}{ Biomarkers of oxidative damage } \\
\hline $\begin{array}{l}\text { Urinary 8-oxodG (ng/mg } \\
\text { creatinine) }\end{array}$ & 3.7 & $2 \cdot 8,4 \cdot 5$ & $3 \cdot 9$ & $3 \cdot 0,4 \cdot 6$ & 3.5 & $2 \cdot 2,3 \cdot 9$ & $3 \cdot 7$ & $2 \cdot 9,4 \cdot 4$ & $17 \cdot 8$ & $31 \cdot 7$ & 0.56 \\
\hline MDA $(\mu \mathrm{g} / \mathrm{l})$ & $9 \cdot 8$ & $8 \cdot 7,11 \cdot 6$ & 11.4 & $10 \cdot 2,12 \cdot 2$ & $10 \cdot 7$ & $9 \cdot 1,12 \cdot 5$ & $11 \cdot 0$ & $9 \cdot 4,13 \cdot 1$ & $17 \cdot 3$ & $20 \cdot 8$ & 0.83 \\
\hline Lipid peroxides $(\mu \mathrm{mol} / \mathrm{l})$ & $195^{\mathrm{a}}$ & 113,248 & $140^{\mathrm{a}}$ & 106,240 & $148^{\mathrm{a}}$ & 99,257 & $85^{\mathrm{b}}$ & 66,183 & 38.7 & $52 \cdot 7$ & 0.73 \\
\hline \multicolumn{12}{|l|}{ Nutrient intakes } \\
\hline Energy (kJ/d) & 10877 & 9688,11811 & 10559 & 9701, 11962 & 9785 & 8537,10714 & 10140 & 9140,13448 & $11 \cdot 6$ & $18 \cdot 7$ & 0.62 \\
\hline Fat $(g / d)$ & 89.1 & $70 \cdot 5,104 \cdot 4$ & $92 \cdot 3$ & $74 \cdot 6,97 \cdot 2$ & $82 \cdot 0$ & $74 \cdot 9,93 \cdot 6$ & 95.4 & $67 \cdot 6,106 \cdot 2$ & $15 \cdot 3$ & $30 \cdot 4$ & 0.50 \\
\hline Carbohydrate (g/d) & 327 & 286,355 & 311 & 271,348 & 278 & 251,331 & 308 & 258,349 & $15 \cdot 0$ & 24.6 & 0.61 \\
\hline Protein $(\mathrm{g} / \mathrm{d})$ & 88.9 & $84 \cdot 8,107 \cdot 9$ & 88.2 & $77 \cdot 6,102 \cdot 5$ & 86.4 & $79 \cdot 0,98 \cdot 2$ & 91.7 & $80 \cdot 9,116 \cdot 1$ & $12 \cdot 7$ & 24.4 & 0.52 \\
\hline Alcohol $(\mathrm{g} / \mathrm{d})$ & 8.7 & $0 \cdot 7,16 \cdot 2$ & 11.8 & $0 \cdot 1,28 \cdot 9$ & 3.7 & $0 \cdot 1,15 \cdot 7$ & $12 \cdot 0$ & $3 \cdot 3,21 \cdot 0$ & $76 \cdot 9$ & $112 \cdot 1$ & 0.69 \\
\hline Vitamin C (mg/d) & 80.1 & $58 \cdot 6,120 \cdot 4$ & 73.9 & $40 \cdot 7,125 \cdot 8$ & 73.0 & $41 \cdot 2,116 \cdot 7$ & $60 \cdot 3$ & $40.8,121.9$ & $35 \cdot 6$ & $60 \cdot 3$ & 0.59 \\
\hline Vitamin E (mg/d) & 8.8 & $7 \cdot 1,9 \cdot 6$ & $7 \cdot 8$ & $6 \cdot 6,10 \cdot 1$ & $10 \cdot 3$ & $7 \cdot 6,10 \cdot 8$ & 8.4 & $6 \cdot 4,10 \cdot 2$ & 21.0 & 33.9 & 0.62 \\
\hline Vitamin A (RE mg/d) & 871 & 713,1450 & 772 & 628,1566 & 1103 & 523,1288 & 1011 & 684,1118 & $37 \cdot 8$ & $103 \cdot 7$ & 0.36 \\
\hline$\beta$-Carotene $(\mathrm{mg} / \mathrm{d})$ & 2445 & 1483,4751 & 2046 & 1340,4493 & 1764 & 937,3683 & 2762 & 1166, 3578 & $52 \cdot 1$ & $75 \cdot 6$ & 0.69 \\
\hline $\begin{array}{l}\text { Fruit and vegetables } \\
\text { (portions/d) }\end{array}$ & $2 \cdot 8$ & $1 \cdot 3,3 \cdot 9$ & $2 \cdot 7$ & $1 \cdot 7,4 \cdot 3$ & $2 \cdot 3$ & $1 \cdot 7,4 \cdot 0$ & $2 \cdot 6$ & $1 \cdot 5,4 \cdot 7$ & $36 \cdot 1$ & $58 \cdot 9$ & 0.61 \\
\hline
\end{tabular}

LQ, lower quartile; UQ, upper quartile; CVintra, intra-individual CV; CVinter, inter-individual CV; AOC, antioxidative capacity, GSH, reduced glutathione, 8-oxodG, 8-oxo-deoxyguanosine; MDA, malondialdehyde; RE, retinol equivalents.

b,c Median values within a row with unlike superscript letters were significantly different $(P<0.05)$

${ }^{*}$ For details of the study protocols, see Materials and methods. 
Table 2. Changes (pre- $v$. post-treatment) of plasma antioxidant concentrations and biomarkers of oxidative damage at $\mathrm{T} 0, \mathrm{~T} 1, \mathrm{~T} 2$ and $\mathrm{T} 3 \dagger$

(Mean values with their standard errors)

\begin{tabular}{|c|c|c|c|c|c|c|c|c|c|}
\hline & \multicolumn{8}{|c|}{ Changes pre- $v$. post-treatment (\%) } & \multirow[b]{3}{*}{$P \ddagger$} \\
\hline & \multicolumn{2}{|c|}{ TO } & \multicolumn{2}{|c|}{$\mathrm{T} 1$} & \multicolumn{2}{|c|}{ T2 } & \multicolumn{2}{|c|}{ T3 } & \\
\hline & Mean & SEM & Mean & SEM & Mean & SEM & Mean & SEM & \\
\hline \multicolumn{10}{|c|}{ Plasma antioxidant concentrations } \\
\hline Vitamin C & 0.3 & 1.8 & $-4 \cdot 4^{*}$ & 1.8 & $-5 \cdot 6^{\star}$ & $1 \cdot 1$ & $-7 \cdot 2^{\star}$ & $2 \cdot 0$ & 0.03 \\
\hline Vitamin E & $1 \cdot 3$ & $2 \cdot 3$ & 4.5 & $2 \cdot 7$ & $3 \cdot 4$ & $2 \cdot 4$ & 5.4 & $2 \cdot 8$ & 0.72 \\
\hline Vitamin A & $2 \cdot 1$ & $2 \cdot 6$ & 5.5 & $3 \cdot 3$ & $4 \cdot 2$ & 3.5 & $4 \cdot 6$ & $2 \cdot 6$ & 0.83 \\
\hline$\beta$-Carotene & -1.4 & $2 \cdot 1$ & -0.3 & 3.0 & $-3 \cdot 1$ & $2 \cdot 2$ & 0.8 & $2 \cdot 3$ & 0.80 \\
\hline AOC & $5 \cdot 0$ & $2 \cdot 1$ & -2.5 & $2 \cdot 8$ & $-6 \cdot 2$ & $2 \cdot 2$ & -3.0 & 2.5 & 0.55 \\
\hline GSH & 1.9 & $3 \cdot 8$ & 0.5 & 4.3 & -0.9 & 2.5 & $-9 \cdot 1$ & 4.0 & 0.54 \\
\hline \multicolumn{10}{|c|}{ Biomarkers of oxidative damage } \\
\hline Urinary 8-oxodG & 0.2 & $4 \cdot 8$ & $6 \cdot 2$ & 4.8 & $24.3^{*}$ & 5.7 & 14.4 & $6 \cdot 3$ & 0.02 \\
\hline MDA & $2 \cdot 8$ & 4.7 & -1.8 & $2 \cdot 8$ & 5.6 & 4.4 & 4.3 & 5.8 & 0.56 \\
\hline Lipid peroxides & $-8 \cdot 2$ & 4.9 & 18.4 & 18.9 & $19 \cdot 2^{*}$ & 9.8 & $25 \cdot 1^{*}$ & 13.5 & 0.02 \\
\hline
\end{tabular}

AOC, antioxidative capacity; GSH, reduced glutathione; 8-oxodG, 8-oxo-deoxyguanosine; MDA, malondialdehyde.

${ }^{*}$ Mean change was significantly from the change pre- $v$. post-treatment at TO $(P<0.05$; Wilcoxon test).

$\dagger$ For details of the study protocols, see Materials and methods.

‡Friedman's ANOVA.

\section{Effect of vitamin intervention plus $100 \%$ oxygen under hyperbaric conditions}

HBO-induced changes of plasma vitamin $\mathrm{C}$ and lipid peroxides were significantly higher compared with changes at T0 (change in pre-T0-post-T0 $v$. change in pre-T3-post-T3; $P<0.05$; Table 2). Except for GSH $(P=0.04)$ HBO-induced changes at $\mathrm{T} 3$ were not statistically different from $\mathrm{HBO}-$ induced changes at $\mathrm{T} 2$ (change in pre-T2-post-T2 v. change in pre-T3-post-T3). There was no correlation between HBO-induced changes of 8-oxodG and lipid peroxides at T2 and $\mathrm{T} 3$, respectively (data not shown).

The individual effect of vitamin $\mathrm{C}$ and $\mathrm{E}$ supplementation on HBO-induced changes in biomarkers of oxidative damage,

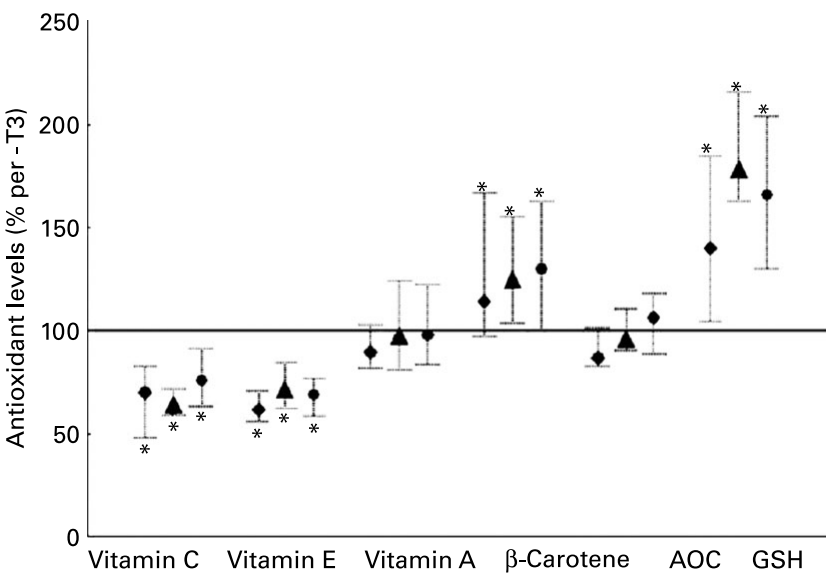

Fig. 2. Changes of blood antioxidants after 4 weeks of supplementation with vitamin $C(500 \mathrm{mg} / \mathrm{d})$ and vitamin $E(165 \mathrm{mg} \alpha$-tocopherol equivalents/d) preT0 $(\diamond)$, pre-T1 $(\boldsymbol{\Delta})$ and pre-T2 $(\bullet)$ in comparison with pre-T3 $(100 \%)$. For details of the study protocols, see Fig. 1 and Materials and methods. Data were analysed with Wilcoxon's test and are shown as medians, with 25-75 percentiles represented by vertical bars. AOC, antioxidative capacity; GSH, reduced glutathione. ${ }^{*}$ Median value was significantly different to that pre-T3 $(P<0.05)$. urinary 8-oxodG excretion and lipid peroxides is shown in Fig. 3. The benefit of the supplementation was limited to $17 \%$ of the volunteers in the case of lipid peroxides and $12 \%$ in the case of urinary 8-oxodG excretion, respectively (Fig. 3). Those volunteers showed an increase in lipid peroxides and urinary 8-oxodG at $\mathrm{T} 2$ above their individual within-subject variance, whereas the HBO-induced changes at T3 were below the within-subject variance. By contrast, 28 and $23 \%$ of the volunteers were affected by an increase in the concentrations of lipid peroxides and urinary 8-oxodG excretion due to HBO after vitamin supplementation (Fig. 3), while their response to $\mathrm{HBO}$ at $\mathrm{T} 2$ was below the within-subject variance. Most of the volunteers (65 and 55\%) did not show any difference between their response to $\mathrm{HBO}$ at $\mathrm{T} 2$ and T3. In terms of 'sensitivity' there was no significant

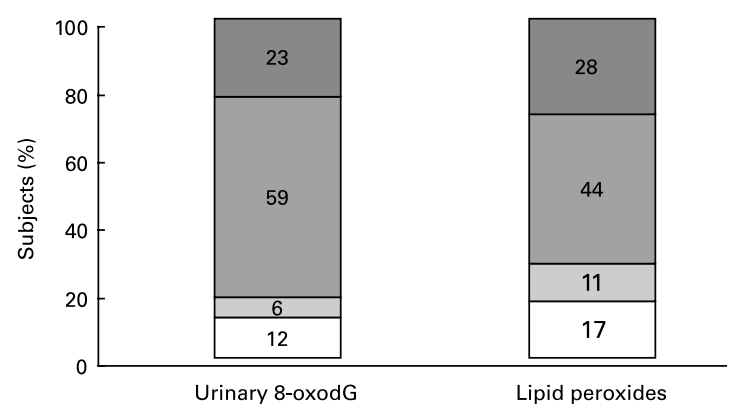

Fig. 3. Distribution of the volunteers ( $n$ 18) according to the effect of hyperbaric $\mathrm{O}_{2}(\mathrm{HBO})$ after supplementation with vitamins $\mathrm{C}$ and $\mathrm{E}$ (T3) on the extent of oxidative damage to DNA and lipids when compared with HBO without vitamin supplementation (T2). For details of the study protocols, see Fig. 1 and Materials and methods. Sensitive subjects (S) were those where $\mathrm{HBO}$-induced changes exceeded twice the square root of the within-subject variance. Non-sensitive subjects (NS) were those where HBO-induced changes did not exceed twice the square root of the within-subject variance. $(\square)$, Enhancement of damage (NS in T2 became S in T3); $(\square)$, no benefit of supplementation ( $\mathrm{S}$ in T2 remained $\mathrm{S}$ in T3); $(\square)$, no influence of supplementation (NS in T2 remained NS in T3); ( $\square$ ), benefit of supplementation (S in T2 became NS in T3); 8-oxodG, 8-oxo-deoxyguanosine. 
change in $\mathrm{HBO}$ response between $\mathrm{T} 2$ and $\mathrm{T} 3$ for both parameters as calculated by McNemar's test (8-oxodG, $P=0 \cdot 68$; lipid peroxides, $P=0 \cdot 72$ ).

At T3 a negative correlation was found for the HBOinduced changes in GSH (pre-T3 $v$. post-T3) and pre-T3 GSH concentrations $(r-0.72 ; P=0.001)$, i.e. those volunteers with high pre-T3 GSH showed smaller HBO-induced changes than those with low pre-T3 GSH concentrations. Similarly, HBO-induced changes in antioxidant capacity (pre-T2 $v$. post-T2) correlated negatively with antioxidant status $(r-0.60 ; P=0.009)$, i.e. those with low antioxidant capacity had the largest change following HBO exposure. Furthermore, the change in 8 -oxodG excretion at T2 correlated negatively with pre-T2 GSH concentration $(r-0.58$; $P=0.013)$. This means that those volunteers with a low GSH status had the largest change following HBO exposure.

\section{Nutrient and antioxidant intake}

The increase of urinary 8-oxodG excretion (pre-T3 v. post-T3) showed a negative correlation with vitamin $\mathrm{E}$ intake from diet ( $r-0.53 ; P=0.023$ ) and from the vitamin supplement $(r-0.53 ; P=0 \cdot 023)$. Change in GSH (pre-T3 $v$. post-T3) was negatively correlated with intake of fruit and vegetables $(r-0.50 ; P=0.043)$. In contrast, at T2 HBO-induced changes of plasma antioxidants and biomarkers of oxidative damage (pre-T2 $v$. post-T2) were not affected by diet $(P>0.05)$.

\section{Discussion}

In the present study HBO exposure was used as a stress model to study the intra-individual and inter-individual consequences of an increased formation of reactive oxygen species on plasma antioxidant concentration as well as biomarkers of oxidative stress in healthy men, and the effects of 4 weeks' supplementation with vitamins $\mathrm{C}$ and $\mathrm{E}$.

When compared with the control situation, a single $2 \mathrm{~h}$ exposure to $\mathrm{HBO}$ decreased plasma vitamin $\mathrm{C}$ and resulted in a significant formation of lipid peroxides and urinary 8-oxodG excretion in comparison with the control situation (Table 2).

The changes observed in plasma vitamin $\mathrm{C}$ were also seen after exposure to $100 \% \mathrm{O}_{2}$ at normobaric conditions (NBO), but, however, without the changes in DNA and lipid oxidation. It thus appears that both $\mathrm{HBO}$ and NBO cause oxidative stress, but that HBO also includes intracellular oxidative stress as evidenced by oxidation of macromolecules. Our findings are in line with the findings of Oter et $a l .{ }^{16}$ demonstrating a directly proportional relationship between oxidative damage and HBO exposure pressure starting from normobaric $100 \%$ exposure.

HBO has been successfully used for the treatment of a variety of clinical conditions related to hypoxia, since $\mathrm{HBO}$ favourably leads to an increase of dissolved $\mathrm{O}_{2}$ in the blood. Narkowicz et al. ${ }^{17}$ were the first supplying evidence for the occurrence of reactive oxygen species in men exposed to HBO. A linear relationship between the formation of $\mathrm{H}_{2} \mathrm{O}_{2}$ and the $\mathrm{O}_{2}$ pressure was shown in rats exposed to $\mathrm{HBO}^{18}$. In a recent experimental study the exposure to $\mathrm{NBO}$ also increased thiobarbituric acid-reactive substance levels in rat lung, brain and blood ${ }^{16}$. Since plasma vitamin $\mathrm{C}$ decreased in the present study, the equilibration between oxidants and antioxidants in the organism seemed to be affected by NBO alone. However, indices of oxidative damage did not increase after exposure to NBO. The present data support the idea that $\mathrm{HBO}$ is a suitable stress model in healthy volunteers. This is in line with previous data ${ }^{19,20}$. When compared with other stress models (for example, smoking, exhausting exercise and chronic diseases), HBO provides the advantage that oxidative stress can be controlled quantitatively.

Supplementation with vitamins $\mathrm{C}$ and $\mathrm{E}$ at five and thirteen times the recommended daily intakes increased plasma vitamins $\mathrm{C}$ and $\mathrm{E}$ by about $30-40 \%$ (Fig. 2) but did not prevent HBO-induced oxidative damage (Table 2). The present study was the first investigating the effect of a combination of vitamins $\mathrm{C}$ and $\mathrm{E}$ on HBO-induced oxidative stress in healthy human subjects. In the present study pharmacological doses of antioxidant vitamins were administered, whereas other authors have used endogenous substances with an antioxidant capacity. The supplementation of human subjects with $\alpha$-lipoic acid reduced HBO-induced lipid and DNA oxidation $^{21}$. In addition a new formulation consisting of wheat gliadin chemically combined with a vegetal preparation of superoxide dismutase prevented the formation of $\mathrm{F}_{2}$-isoprostanes and DNA strand breaks due to HBO in human subjects $^{22}$.

It is important to point out that antioxidant supplements are not always safe. Although toxicity of consumed antioxidants is very low and only occurs at very high intake levels ${ }^{23}$, the degree of benefit or harm of antioxidant supplementation depends also on genetic susceptibilities. Besides that it is by no means clear how antioxidant supplements interact with each other and with other dietary constituents affecting the in vivo redox balance. There are a few studies showing that individual genetic polymorphisms related to the activity of metabolic and detoxification enzymes influence the effects of antioxidants. For example, it was shown that differences in base excision repair capacity which is due to polymorphisms in the XRCC1 gene may modulate the effect of dietary antioxidant intake on prostate cancer risk $^{24}$. Antioxidants and oxidants are able to activate certain genes and signalling pathways by modulating the redox state of the cell. It was also shown in cultured cells that vitamin $\mathrm{C}$ might affect gene expression and this seems to be mediated by its redox state.

To our knowledge there are no studies investigating the influence of antioxidant intake and basal antioxidants on NBO- and HBO-induced oxidative damage. We found that NBO-induced changes were not affected by the intake of antioxidants or by the basal antioxidant status. HBO-induced changes of antioxidant capacity showed a negative association with basal concentration. This indicates a greater decrease in plasma antioxidants in human subjects with lower basal levels, i.e. individuals with lower antioxidant status are more vulnerable to oxidative stress. Accordingly, a low basal GSH concentration at T2 resulted in more damage to DNA as indicated by a higher urinary 8-oxodG excretion. However, these associations were not seen at T3. Since basal GSH concentrations were markedly smaller at T3 we expected an even stronger association to urinary 8-oxodG excretion at T3. It can only be supposed that the real effects were masked by the effects of the excessive antioxidant supplementation. 
While the intake of antioxidants did not seem to have any influence on changes due to NBO and HBO, two correlations were found to be of significance due to $\mathrm{HBO}$ plus vitamin supplementation. First, a higher intake of vitamin $\mathrm{E}$ correlated with a lower 8-oxodG concentration in urine. The source of vitamin E (diet or supplement) made no difference. Second, the negative correlation between fruit and vegetable intake and HBO-induced changes in GSH concentration suggests that a higher intake of fruit and vegetables 'saved' the consumption of GSH. Discrepancies in the results at T2 and T3, respectively, might be explained by the vitamin intervention before $\mathrm{HBO}$ at $\mathrm{T} 3$, potential genetic differences between individuals and thus their susceptibility as well as by the intra-individual differences (Table 1) resulting from the longitudinal protocol of the present study. The chosen study design has a lack of strength since there is no parallel arm and there is no sufficient evidence that differences between the treatments may derive from chronic prolonged oxidative stress as a result of the acute stress.

In the steady state, urinary excretion of 8-oxodG in principle reflects the rate of oxidative damage, whereas the level of lesions from surrogate cells should reflect the balance between damage and repair. Although the volunteers were asked to avoid physical, mental and environmental stress before treatments, increased 8-oxodG concentrations in urine might also be due to the repair of pre-existing damage.

The present study was on healthy and young volunteers and the findings may not be representative of any nutritional or health compromise. Moreover, intervention data showed that supplementation-induced changes of plasma vitamin $\mathrm{C}$ and $E$ levels were also characterised by a high variation inter-individually ranging from 'non-responders' up to increases by 133 and $107 \%$, respectively. However, the broad range of response to supplementation was not correlated to the HBOinduced formation of biomarkers of oxidative stress. Although the compliance was assessed to be $92 \%{ }^{25}$, devious non-compliant volunteers which influence the assessment result might not be identified. Besides that, individual response to vitamin supplementation depends mainly on metabolic rate, tissue distribution and other related factors, where genetic differences may have an impact. In order to explain discrepancies in the individual response, and thus their susceptibility, future research might be focused on genetic differences.

The homeostasis of the antioxidative network might mainly explain the decline of the two parameters as described for vitamin $\mathrm{C}$ and uric acid ${ }^{26}$. Besides type and dosage of the supplementation in the present study, pro-oxidative activities of the supplementation might also be responsible for the lack in prevention of the HBO-induced oxidative damage. This is supported by the decrease in GSH after supplementation. After all, it cannot be ruled out that the chosen antioxidants were effective in the prevention of HBO-induced damage, since it is possible that various types of antioxidants exert different effects that are detected by different endpoints. It is also suggested that some of the biomarkers of oxidative stress and total antioxidant capacity are insufficiently validated in vivo and therefore not suitable for such investigations ${ }^{27}$. As an example, antioxidant capacity has failed to demonstrate an effect of supplementation with antioxidants or antioxidant-rich foods in human subjects and is therefore considered as a biomarker with limited value in vivo ${ }^{28}$. Since we did not use the parameter of antioxidant capacity in isolation but with other well-validated biomarkers of oxidative stress, there is nothing to be said against its usage in the present study.

A low fruit and vegetable intake is one of the three most important behavioural and environmental risk factors of death cause in low- and middle-income countries ${ }^{29}$. Whilst the risk factor exists in these countries it is not relevant in a wealthy population. With respect to that finding, the lack in the present study of finding a positive effect of antioxidant intervention might be covered by factors resulting from a high body fat mass and a good nutritional status which is characteristic for high-income countries. Since the volunteers of the present study had a good nutritional status and were not underweight, this may add to our effects.

In summary, $131 \mathrm{~min}$ of breathing $\mathrm{O}_{2}$ under pressure of $240 \mathrm{kPa}$ induces oxidative stress in healthy volunteers as evidenced by an increase in urinary 8-oxodG excretion and plasma lipid peroxides with concomitant decreases in plasma vitamin C. Oxidative stress was not prevented by 4 weeks' supplementation with vitamins $\mathrm{C}$ and $\mathrm{E}$. There were high intra- and inter-individual variances in biomarkers tested. Intra- and inter-individual differences of HBO-induced oxidative stress were neither explained by antioxidant intake nor by basal antioxidative status of the volunteers.

\section{Acknowledgements}

The work was funded by the Graduiertenkolleg 820 'Natural antioxidants - their occurrence in plants, food, animals and humans' of the German Research Foundation (DFG). The vitamin $\mathrm{C}$ and $\mathrm{E}$ supplement CellaVie was a gift from Ferrosan A/S (Denmark). The authors thank Ferrosan A/S, the staff of the German Naval Medical Institute Kronshagen, and most of all the volunteers who made the present study possible.

\section{References}

1. Ames BN, Gold LS \& Willett WC (1995) The cause and prevention of cancer. Proc Natl Acad Sci U S A 92, 5258-5265.

2. Sies H (1997) Antioxidants in disease mechanism and therapy. In Advances in Pharmacology, pp. 1-691 [H Sies, editor]. San Diego and London: Academic Press.

3. Blot WJ, Li JY, Taylor PR, et al. (1993) Nutrition intervention trials in Linxian. China: supplementation with specific vitamin/ mineral combinations, cancer incidence, and disease-specific mortality in the general population. J Natl Cancer Inst 85, $1483-1492$.

4. Hennekens CH, Buring JE, Manson JE, et al. (1996) Lack of effect of long-term supplementation with $\beta$ carotene on the incidence of malignant neoplasms and cardiovascular disease. $N$ Engl J Med 334, 1145-1149.

5. Stephens NG, Parsons A, Schofield PM, Kelly F, Cheeseman K \& Mitchinson MJ (1996) Randomized, controlled trial of vitamin $\mathrm{E}$ in patients with coronary disease. Cambridge Heart Antioxidant Study (CHAOS). Lancet 347, 781-786.

6. Salonen JT, Nyyssonen K, Salonen R, et al. (2000) Antioxidant supplementation in atherosclerosis prevention (ASAP) study: a randomized trial of the effect of vitamin $\mathrm{E}$ and $\mathrm{C}$ on 3-year progression of carotid atherosclerosis. J Intern Med 248, $377-386$.

7. van den Berg R, van Vliet T, Broekmans WM, Cnubben NH, Vaes WH, Roza L, Haenen GR, Bast A \& van den Berg H (2001) A vegetable/fruit concentrate with high antioxidant 
capacity has no effect on biomarkers of antioxidant status in male smokers. J Nutr 131, 1714-1722.

8. Heart Protection Study Collaborative Group (2002) MRC/BHF Heart Protection Study of antioxidant vitamin supplementation in 20536 high-risk individuals: a randomised placebo-controlled trial. Lancet 360, 23-33.

9. Möller P, Vogel U, Pedersen A, Dragsted LO, Sandstrom B \& Loft S (2003) No effect of 600 grams fruit and vegetables per day on oxidative DNA damage and repair in healthy nonsmokers. Cancer Epidemiol Biomarkers Prev 12, 1016-1022.

10. Dragsted LO, Pedersen A, Hermetter A, et al. (2004) The 6-aday study: effects of fruits and vegetables on markers of oxidative stress and antioxidative defense in healthy nonsmokers. Am J Clin Nutr 79, 1060-1072.

11. Thompson HJ, Heimendinger J, Gillette C, Sadlacek SM, Haegele A, O'Neill C \& Wolfe P (2005) In vivo investigation of changes in biomarkers of oxidative stress induced by plant food rich diets. J Agric Food Chem 53, 6126-6132.

12. Miller ER III, Pastor-Barriuso R, Dalal D, Riemersma RA, Appel LJ \& Guallar E (2005) Meta-analysis: high-dosage vitamin E supplementation may increase all-cause mortality. Ann Intern Med 142, 37-46.

13. Möller P \& Loft S (2002) Oxidative DNA damage in human white blood cells in dietary antioxidant intervention studies. Am J Clin Nutr 76, 303-310.

14. Weimann A, Belling D \& Poulsen HE (2002) Quantification of 8-oxo-guanine and guanine as the nucleobase, nucleoside and deoxynucleoside forms in human urine by high-performance liquid chromatography-electrospray tandem mass spectrometry. Nucleic Acids Res 30, E7.

15. Bartels H \& Bohmer M (1971) Micro-determination of creatinine. Clin Chim Acta 32, 81-85.

16. Oter S, Korkmaz A, Topal T, Ozcan O, Sadir S, Ozler M, Ogur B \& Bilgic H (2005) Correlation between hyperbaric oxygen exposure pressures and oxidative parameters in rat lung, brain, and erythrocytes. Clin Biochem 38, 706-711.

17. Narkowicz CK, Vial JH \& McCartney PW (1993) Hyperbaric oxygen therapy increases free radical levels in the blood of humans. Free Rad Res Commun 19, 71-80.

18. De Martino G, Luchetti M \& De Rosa RC (1996) Toxic effects of oxygen. In Handbook on Hyperbaric Medicine, pp. 59-74 [G Oriani, A Marroni and F Wattel, editors]. New York: Springer-Verlag.

19. Speit G, Dennog C \& Lampl L (1998) Biological significance of DNA damage induced by hyperbaric oxygen. Mutagenesis 13, 85-87.

20. Benedetti S, Lamorgese A, Piersantelli M, Pagliarani S, Benvenuti F \& Canestrari F (2004) Oxidative stress and antioxidant status in patients undergoing prolonged exposure to hyperbaric oxygen. Clin Biochem 37, 312-317.

21. Alleva R, Nasole E, Di Donato F, Borghi B, Neuzil J \& Tomasetti M (2005) $\alpha$-Lipoic acid supplementation inhibits oxidative damage, accelerating chronic wound healing in patients undergoing hyperbaric oxygen therapy. Biochem Biophys Res Commun 333, 404-410.

22. Muth CM, Glenz Y, Klaus M, Radermacher P, Speit G \& Leverve X (2004) Influence of an orally effective SOD on hyperbaric oxygenrelated cell damage. Free Rad Res 38, 927-932.

23. Bendich A \& Machlin LJ (1988) Safety of oral intake of vitamin E. Am J Clin Nutr 48, 612-619.

24. van Gils CH, Bostick RM, Stern MC \& Taylor JA (2002) Differences in base excision repair capacity may modulate the effect of dietary antioxidant intake on prostate cancer risk: an example of polymorphisms in the XRCC1 gene. Cancer Epidemiol Biomarkers Prev 11, 1279-1284.

25. Bader N, Bosy-Westphal A, Koch A \& Mueller MJ (2006) Influence of vitamin $\mathrm{C}$ and $\mathrm{E}$ supplementation on oxidative stress induced by hyperbaric oxygen in healthy men. Ann Nutr Metab 50, 173-176.

26. Huang HY, Appel LJ, Choi MJ, Gelber AC, Charleston J, Norkus EP \& Miller ER III (2005) The effects of vitamin C supplementation on serum concentrations of uric acid: results of a randomized controlled trial. Arthritis Rheum 52, 1843-1847.

27. Verhagen H, Bujisse B, Jansen E \& Bueno-de-Mesquita B (2006) The state of antioxidant affairs. Nutr Today 41, 244-250.

28. Griffiths HR, Moller L, Bartosz G, et al. (2002) Biomarkers. Mol Aspects Med 23, 101-208.

29. Danaei G, Vander Hoorn S, Lopez AD, Murray CJ \& Ezzati M; Comparative Risk Assessment Collaborating Group (Cancers) (2005) Causes of cancer in the world: comparative risk assessment of nine behavioural and environmental risk factors. Lancet 366, 1784-1793. 